\section{Development and Disability}

\section{G24 A CLINICAL EVALUATION OF VISION ASSESSMENT IN CHILDREN WITH SENSORI-NEURAL DEAFNESS}

R. Guy, J. Nicholson, S.S. Pannu, R. Holden. Ronnie MacKeith Child Development Centre, Derbyshire Childrens Hospital, Derby, UK

Since 1991, children with sensori-neural deafness and their families in the Southern Derbyshire Health Authority have been supported by a holistic multi-agency approach to their diagnosis and management.

Analysis of 122 children assessed at the Child Development Centre has shown a high number with additional vision problems. The ages of the children ranged from 8 months to 16 years, and 72 had newly diagnosed sensori-neural deafness, the majority under 5 years. Of these children, 66 had congenital sensori-neural deafness associated in 15 with specific syndromes, 3 being related to vision. Eleven had acquired deafness, and there were 45 of unknown cause. The severity of deafness varied from $40 \mathrm{~dB}$ in the better ear to profound deafness. Of 110 children whose vision has been fully assessed, $48(43.6 \%)$ had ocular abnormalities. These included a high number $(40.9 \%)$ of refractive errors, ranging from mild to severe, and also 6 cases of Usher syndrome, mainly type 2 . Some children had more than one eye defect.

The reported findings are that deaf and hearing impaired children are three times more likely to develop ocular abnormalities than their hearing peers, which makes early detection of paramount importance.

An important outcome has been the establishment of local guidelines for both vision assessent and screening for Usher syndrome by electrophysiological testing at aged 7 years and above. Screening has been completed to date in 71 children with congenital sensori-neural deafness. More research is needed to address ethical issues of screening for Usher syndrome and to establish national clinical guidelines.

\section{G25 TELOMERE TESTING IN CHILDREN WITH SEVERE LEARNING DIFFICULTIES}

D. Cody, P.J. Seymour. Wirral Services for Child Health, UK

Background and aims: Recent advances in cytogenetic techniques to detect submicroscopic chromosome rearrangements involving telomeres have refocused attention on children with severe learning difficulties of unknown aetiology. The aim of our study was to investigate the usefulness of telomere analysis in such children.

Methods: A casenote review of the 83 children attending Stanley school for children with severe learning difficulties on the Wirral was carried out and those with no diagnosis were ascertained. Previous investigations in these children, including karyotype, were negative. Those with accompanying dysmorphic features and / or a positive family history of chromosomal anomalies were selected. Blood samples were taken from these for telomere analysis using the Vysis Totel Vision probe kit.

Results: 16 of the children (19\%) attending the school had no diagnosis. 9 of these $(11 \%)$ fitted our criteria for telomere analysis. Consent for blood testing was obtained from 8 of this group. Fluorescence in situ hybridisation studies using telomere probes for all the autosomes and sex chromosomes were carried out and revealed no evidence of deletion or rearrangement of any of the telomeric regions in this group.

Conclusions: Despite applying selective criteria no positive results were obtained from this small sample. However the importance of a diagnosis for families and thus more accurate genetic counseling should not be underestimated and needs to be balanced against the cost ( $£ 150$ per test) and the low yield from this test.

\section{G26 A PROSPECTIVE, POPULATION BASED ASSESSMENT OF DEVELOPMENTAL DELAY AND CRANIOFACIAL DYSMORPHISM IN CHILDREN BORN TO MOTHERS WITH EPILEPSY}

F.H. James, U.K. Wariyar, J. Burn, S.A. Lynch. Department of Human Genetics, Newcastle upon Tyne, UK

Children of women with epilepsy are at increased risk of developmental delay, craniofacial dysmorphism \& malformation.
Aims: 1) To determine the true incidence of developmental delay in our prospectively recruited, non-hospital based cohort of children aged $2-3$ years. 2) To identify the aetiological risk factors involved. 3) To establish whether delay occurs independently of malformation and craniofacial dysmorphism.

Methods: One hundred and eleven children have been assessed. The Bayley's scales are used to assess Mental Developmental Index (MDI). Facial measurements are taken with calipers and clinical photographs obtained.

Results: Of 111 children, 88 had normal development (79\%) and 23 delayed performance $(21 \%),(\mathrm{p}=0.0215)$. The proportion with severe delay (MDI $>2$ SDs below mean), $(8 / 111=7.2 \%)$ is substantially greater than the background rate of $1.5 \%$, and is highly statistically significant $(\mathrm{p}=0.0006)$. We found a significant relationship between developmental delay and poor maternal educational attainment, $(\mathrm{p}=0.0118)$. There also appears to be significant correlation between developmental delay \& in utero exposure to CBZ $(\mathrm{p}=$ $0.0340)$, and VPA polytherapy $(\mathrm{p}=0.0049)$. Seizures in pregnancy are unrelated to developmental delay $(p=0.453)$. We have found no correlation between dysmorphism, malformation \& developmental delay. This is irrespective of drug dose.

Conclusions: Children born to women with epilepsy are at significantly increased risk of developmental delay. This may be associated with maternal intelligence $\&$ certain anticonvulsants. It occurs independently of malformation \& craniofacial dysmorphism.

\section{G27 ARE WE STILL LATE IN PICKING UP DUCHENNE MUSCULAR DYSTROPHY?}

I. Anand, V. Rao. Walsall Community Health Trust, UK

Objective: To identify the time interval between presentation of symptoms and confirmation of diagnosis of Duchenne muscular dystrophy (DMD).

Design: Retrospective review of community and hospital medical records of children diagnosed with DMD.

Setting: Walsall Health District of West Midlands, with population of 60,000 children.

Method: All cases of DMD currently being cared for in Walsall were identified, and records reviewed.

Results: There were 7 boys with the diagnosis of DMD. (1) Two of the cases were identified after DMD was diagnosed in siblings. (2) Confirmation of DMD in two siblings led to the termination of an affected foetus. (3) The average of presentation was $28.4 \mathrm{mths}(6 \mathrm{~m}$ to $49 \mathrm{~m})$. Presenting symptoms were waddling gait $(\mathrm{n}=2)$, frequent falls $(n=1)$, speech delay $(n=1)$, difficulty in climbing stairs $(n=1)$. (4) In 3 cases limited abduction of hips in early infancy led to hospital referral. However this did not directly lead to the suspicion and diagnosis of DMD. (5) Average age of confirmation of diagnosis was $47.6 \mathrm{mths}$ $(12 \mathrm{~m}$ to $68 \mathrm{~m})$. (6) Average delay in the confirmation of diagnosis was 19.2 months ( 6 months to 28 months).

Conclusion and remarks: (1) There is great diversity in the presenting symptoms of DMD. (2) Limited abduction of the hips in early infancy may be an early presentation of DMD. (3) None presented with late walking (over 18 months). (4) There continues to be a significant delay between the onset of symptoms and the confirmation of diagnosis of DMD.

\section{G28 COGNITIVE IMPAIRMENT IN 6-8 YEAR OLD CHILDREN FOLLOWING NEONATAL ENCEPHALOPATHY}

C.E. Rands, A.S. Rose, N. Marlow, E.S. Draper ${ }^{1}$. Division of Child Health, School of Human Development, University of Nottingham $;{ }^{1}$ Department of Epidemiology \& Public Health, University of Leicester, UK

Aims: Few studies have evaluated outcome for children without disability following neonatal encephalopathy (NE). We have performed a regionally-based study of outcome in middle childhood for such a group. NE was defined as moderate or severe encephalopathy thought to be due to hypoxia before birth after 35 weeks gestation.

Methods: Children born between 1992-94 were identified from the Trent regional database and examined at school with a matched classmate as a control, using the British Ability Scales 2e and NEPSY.

Results: 45 cases have been evaluated between 78 and 95 months of age. Of these, $7(16 \%)$ were attending special schools and were not matched to controls; 6 had cerebral palsy (4 quadriplegia) and 3 had multiple disabilities. Scores for the remaining children are shown in the table. 
Abstract G28

\begin{tabular}{|c|c|c|c|c|}
\hline & & \multirow[b]{2}{*}{ Control } & \multicolumn{2}{|c|}{ Encephalopathy } \\
\hline & & & Not ventilated & Ventilated \\
\hline Mainstream school n & 34 & 21 & 17 & \\
\hline \multirow{5}{*}{ BAS $2 \mathrm{e}-$} & GCA median & 114.5 & 108.5 & $104^{\star}$ \\
\hline & Spatial & 107 & 99 & $92^{\star}$ \\
\hline & Verbal & 118.5 & 118 & $113^{\star}$ \\
\hline & Pictorial & 110 & 104.5 & $101^{\star}$ \\
\hline & Reasoning & & & \\
\hline \multirow[t]{4}{*}{ NEPSY- } & Attention & 116 & 108.5 & $104^{\star}$ \\
\hline & Executive & & & \\
\hline & Memory & 103.5 & 105 & $88^{\star}$ \\
\hline & Language & 123 & 110.5 & $104^{\star}$ \\
\hline
\end{tabular}

${ }^{\star} \mathrm{p}<0.05$ compared to controls (Wilcoxon rank sum test).

In this population there was a gradation of performance across the 3 groups. The ventilated NE group consistently scored lower compared to controls.

Conclusions: Children who survive moderate or severe encephalopathy without disability are at risk of later neuro-cognitive dysfunction and out-come appears to relate to the severity of neonatal condition.

\section{G29 HOW PARENTS EXPERIENCE MULTI-DISCIPLINARY MEETINGS ABOUT THEIR CHILD}

C. Burton, E. Knight-Jones. Nottingham Child Development Centre, UK

From the 1980s parents have taken part in the CDC assessment coordinating meetings and are now routinely invited. Staff had concerns that this was inevitably a stressful experience for any parent. This study aims to learn about parents' views and experiences of ACMs, find out if they are useful for parents and identify ways to make the assessment process more 'parent friendly'.

All children whose parents had attended multi-disciplinary meetings from January 1999 to March 2000 were included (57 children). Parents were asked to complete a questionnaire over the telephone and this was anonymised. 48 were completed. The questionnaire contained mainly closed questions with open questions relating to parents' experiences and views of the meeting.

$94 \%$ of parents were happy with the assessment process and surprisingly only $40 \%$ of parents found the meeting stressful. 45 out of 48 questioned understood the reason for their child seeing the professionals involved and all but one were glad they had attended. The results showed that most parents are happy with the coordinating meeting and assessment process.

However, many important suggestions have been made, in particular the use of jargon needs to be limited and the layout of the meeting room and seating of professionals and parents needs improvement. It was also suggested that parents receive leaflets explaining what will happen at the meeting (these are now provided).

\section{G30 TOWARDS NATIONALLY USEFUL DEFINITIONS OF DISABILITY}

T. Hutchison, R. Black, D. Gordon, H. Leich, R. Mallett, P. Stallard. Child Health Department, Bath, UK

Aims: To adapt methodology from the 1986 OPCS survey of disability in GB, to produce a tool for doctors teachers and social workers to use with parents to define disability for the purpose of registration.

Methods: We selected 189 children aged 5-15 randomly from the district disability register. Doctors who did not know the children analyzed their medical records. A research assistant interviewed the first 100 consenting families using a structured questionnaire. Parents and doctors were asked about presence and severity of disability in 10 categories. Parents were asked detailed questions about the characteristics of their children in each category. OPCS descriptors of severity were used except in the case of behavior and learning. Here the General Health and Behavior Questionnaire (GHBQ) and National Curriculum Standardized Attainment Targets (SATs) were substituted.

Results: There was high correlation between parents and doctors about numbers and type of disability, but doctors often gave a lower assessment of severity. OPCS descriptors with their hierarchy of severity were validated for mobility, hand function, continence and communication. The GHBQ had a strong correlation with parental perception of behavior disability. Parental assessment of learning disability had a strong correlation with SATs descriptors. Hearing and vision had the worst correlation between doctors and parents because of ambiguity about measurement of impairment rather than disability. The OPCS hierarchy of severity of self-care descriptors was not valid for children.

Conclusions: We now have disability registration guidelines for doctors and social workers, validated against parent's perceptions of severity. More work is needed on under fives, self-care, and the usefulness of SATs as a measure of learning disability.

\section{G31 AUDIT OF AN ADHD CLINIC WITH PARTICULAR REFERENCE TO THE USE OF RISPERIDONE FOR ASSOCIATED OPPOSITIONAL DEFIANT DISORDER AND CONDUCT DISORDER}

G.D. Kewley. Learning Assessment Centre, W Sussex, UK

Audit of a clinic population regarding diagnosis and management, particularly 104 children with associated Oppositional Defiant Disorder (ODD)/Conduct Disorder (CD). Additionally the clinic's use of risperidone was analysed. The children attended a multidisciplinary clinic with a national referral basis, specialising in neurodevelopmental and learning problems, especially ADHD and related conditions.

$64 \%$ had combined ADHD and of these $57 \%$ had associated early onset ODD and/or CD. $73 \%$ of this latter group were not adequately treated by Ritalin or Dexedrine alone, requiring a second medication-generally initially clonidine-to achieve a $92 \%$ very positive outcome. 30 children aged 6-21 years were treated with risperidone, usually because of persistent $\mathrm{ODD} / \mathrm{CD}$. Interval between initial diagnosis and institution of risperidone treatment, between 0 months and 6 years. Dosage: between $0.5 \mathrm{mg}$ and $6 \mathrm{mg}$ daily. $50 \%$ required a bd dosage. Risperidone treatment duration in these patients - up to 6 years. 25 (67\%) showed moderate or very significant improvement. In 5, risperidone was stopped because of no improvement (2) or excessive weight gain (3). Other side effects: vomiting, drowsiness or withdrawal dyskinesia (1). Liver function tests were normal.

Preliminary data suggest significant improvement can be achieved in children with ADHD and comorbid early onset ODD/CD by more intensive management, often with more than one medication. Risperidone may have a significant place in the management of these children where other treatments have proved unsuccessful. 